\title{
Transcatheter Tricuspid Valve-In-Ring and Aortic Valve-In-Valve Implantation
}

\author{
Daniel Reichart ${ }^{1} \quad$ Niklas Schofer ${ }^{2}$ Florian Deuschl $^{2}$ Andreas Schaefer ${ }^{1}$ Stefan Blankenberg ${ }^{2}$ \\ Hermann Reichenspurner ${ }^{1}$ Ulrich Schaefer ${ }^{2}$ Lenard Conradi ${ }^{1}$
}

\author{
1 Department of Cardiovascular Surgery, Universitätsklinikum \\ Hamburg-Eppendorf, Universitäres Herzzentrum Hamburg GmbH, \\ Hamburg, Germany \\ 2 Department of General and Interventional Cardiology, \\ Universitätsklinikum Hamburg-Eppendorf, Universitäres \\ Herzzentrum Hamburg GmbH, Hamburg, Germany
}

Thorac Cardiovasc Surg Rep 2017;6:e29-e31.

\author{
Address for correspondence Daniel Reichart, MD, \\ Universitätsklinikum Hamburg-Eppendorf, Universitäres \\ Herzzentrum Hamburg GmbH, Martinistr. 52, 20246 Hamburg, \\ Germany (e-mail: d.reichart@uke.de).
}

\begin{abstract}
Keywords

- aortic valve

- tricuspid valve

- heart valve

Background Transcatheter heart valve (THV) therapies have shown to be an alternative to surgical valve replacement, especially in high-risk patients requiring redo surgery. However, reports of transcatheter-based interventions in tricuspid valve position are scarce.

Case Description Here, we report a case of successful concomitant transcatheter aortic valve-in-valve (ViV) and tricuspid valve-in-ring (ViR) procedures using a 23-mm CoreValve Evolut R THV (Medtronic, Inc., Minneapolis, Minnesota, United States) in aortic position and a 29-mm SAPIEN3 (Edwards Lifesciences, Inc., Irvine, California, United States) THV in tricuspid position.

Conclusion This case demonstrates feasibility of concomitant transcatheter aortic ViV and tricuspid ViR procedures.
\end{abstract}

\section{Introduction}

Reports on transcatheter tricuspid valve interventions are scarce, even more when combined with other valve procedures. However, potential candidates are abundant and thus, this case report faces the clinical need and reports on an alternative therapeutic option of concomitant transcatheter aortic valve-in-valve (ViV) and off-label tricuspid valve-in-ring (ViR) implantation. ${ }^{1,2}$

\section{Case Description}

A 77-year-old female patient was referred with dyspnea according to New York Heart Association (NYHA) functional class, III-IV, 22 months after aortic valve replacement (Hancock II Ultra 21 mm, Medtronic, Inc., Minneapolis, Minnesota, United States). During this initial procedure, right coronary obstruction occurred with subsequent surgical revascularization on day 1 . Nonetheless, right heart dysfunction and severe tricuspid regurgitation (TR) developed requiring tricuspid valve repair 3 months later using a 34-mm Contour 3D ring (Medtronic, Inc.). Nine months later, the patient was admitted with infective aortic valve endocarditis, which was treated with antibiotics. Subsequent early deterioration of the aortic bioprosthesis was possibly triggered by this event. Upon readmission another 13 months later, severe aortic valve regurgitation without any sign of active endocarditis was seen. The peak/mean transprosthetic gradients were 59/33 mm Hg. Left ventricular ejection fraction was $43 \%$ and right ventricular function was severely reduced (tricuspid annular plane systolic excursion, $6 \mathrm{~mm}$ ) with a recurrence of severe TR and mild chronic renal failure. The ventricular pacemaker (PM) lead was located at the interrupted portion of the annuloplasty ring. Formal risk stratification revealed logistic European System for Cardiac Operative Risk Evaluation (logEuroSCORE) I of 34.4\% and Society of Thoracic Surgeons (STS) score of $10.5 \%$. The patient was allocated to a combined interventional treatment due to prohibitive surgical risk. Use of a 23-mm CoreValve Evolut R THV (Medtronic, Inc.) as an aortic ViV procedure and a 29-mm SAPIEN3 (Edwards Lifesciences, Inc., Irvine, California, received

March 31, 2017

accepted after revision

July 19, 2017
DOI https://doi.org/

10.1055/s-0037-1606345. ISSN 2194-7635. (c) 2017 Georg Thieme Verlag KG Stuttgart · New York

\section{License terms}

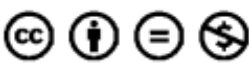


United States) THV as a tricuspid ViR procedure was planned. The dimensions provided by the manufacturer of the Medtronic Hancock II Ultra $21 \mathrm{~mm}$ were: internal diameter (ID), $18.5 \mathrm{mmand}$ true stent ID, $16.5 \mathrm{~mm}$. The $34-\mathrm{mm}$ Contour 3D ring had a $\mathrm{min} / \mathrm{max}$ diameter of $22 / 32 \mathrm{~mm}$. The orifice area was $588.6 \mathrm{~cm}^{2}$ resulting in an area-derived inner diameter of $27.4 \mathrm{~mm}$. By CT, inner ring perimeter of $87.6 \mathrm{~mm}$ and perimeter-derived diameter of $27.9 \mathrm{~mm}$ were measured.

The procedure was performed under general anesthesia using ProStar (right common femoral artery; Abbott Laboratories, Chicago, Illinois, United States) and ProGlide systems (right femoral vein; Abbott Laboratories). A transradial cerebral protection system (Claret Medical, Santa Rosa, California, United States) was introduced. Due to low left coronary takeoff (distance, $6 \mathrm{~mm}$ ), prewiring of the left coronary system using a balance middle weight (BMW) wire (Abbott Laboratories) and $3.0 \times 20 \mathrm{~mm}$ percutaneous coronary intervention $(\mathrm{PCI})$ balloon (Boston Scientific, Inc., Marlborough, Massachusetts, United States) was performed.

First, the deteriorated aortic bioprosthesis was passed retrogradely, and a 260-cm Safari wire (Boston Scientific, Inc.) was introduced into the left ventricle. Due to the small caliber peripheral vasculature, the 23-mm CoreValve Evolut R THV (Medtronic, Inc.) was inserted using the $14 \mathrm{Fr}$ equivalent InLine sheath (Medtronic, Inc.). Aortic ViV procedure was performed without rapid ventricular pacing (RVP) or resheathing. Fluoroscopy, echocardiography (peak/mean gradient: $17 / 8 \mathrm{~mm} \mathrm{Hg}$ ), and invasive hemodynamic (peak/ mean gradient: $12 / 2 \mathrm{~mm} \mathrm{Hg}$ ) assessment confirmed adequate THV position without any paravalvular leakage (PVL).

In a second step, tricuspid ViR was performed. A 29-mm SAPIEN3 THV (Edwards Lifesciences, Inc.) was introduced using a 300 -cm Safari wire (Boston Scientific, Inc.), 16Fe-Sheath, and Commander delivery catheter (both from Edwards Lifesciences, Inc.) with reverse crimping to account for the antegrade approach ( $\mathbf{- F i g . 1}$ ). Loading of the THV onto the balloon was performed in the right atrium with balloon overfilling by $2 \mathrm{~mL}$ due to marginal landing zone dimensions. THV deployment resulted in an implantation height of $80 \%$ ventricular (-Fig. 2). Procedure was performed under fast pacing of 150

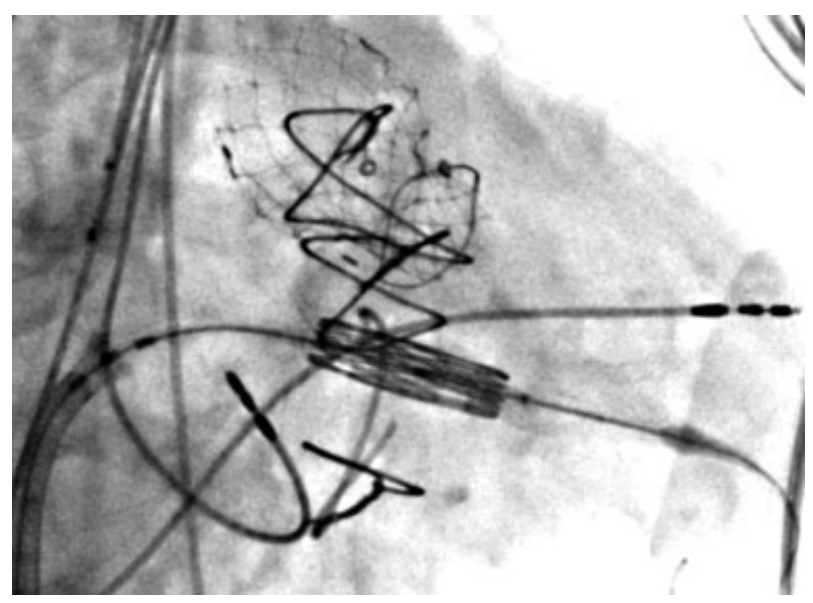

Fig. 1 The CoreValve Evolut R THV (Medtronic, Inc.) was implanted in aortic position. The 29-mm SAPIEN3 THV (Edwards Lifesciences, Inc.) was introduced with reverse crimping to account for the antegrade approach.

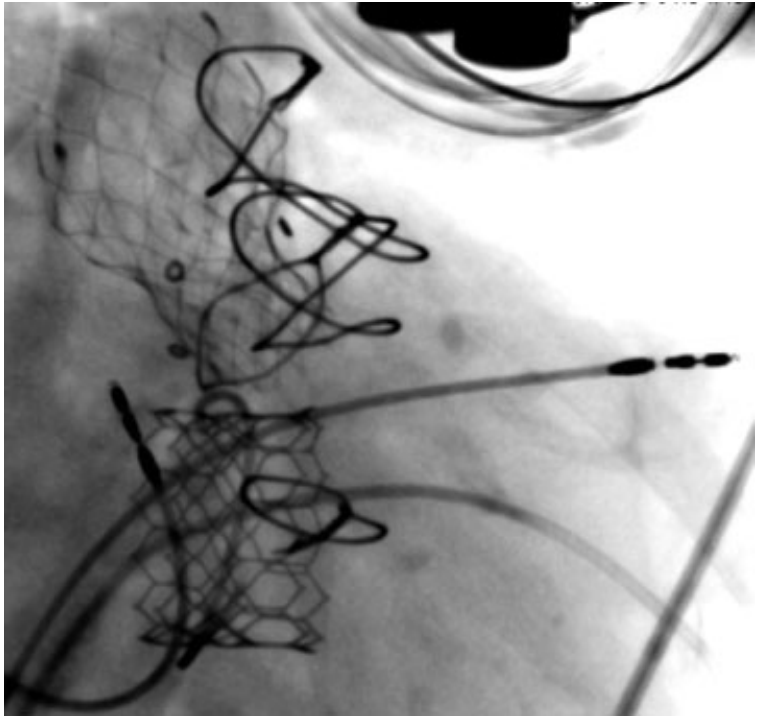

Fig. 2 Postimplantation fluoroscopy depicting the SAPIEN3 (Edwards Lifesciences, Inc.) THV in tricuspid position and a CoreValve Evolut R THV (Medtronic, Inc.) in aortic position. The SAPIEN3 (Edwards Lifesciences, Inc.) was implanted with an implantation height of $80 \%$ into the ventricle.

beats per minute via external programmer and internal PM. Balloon postdilatation was performed using a 30-mm Z-MED II balloon (NuMED; Hopkinton, New York, United States).

Procedural/fluoroscopy times were 163/56 minutes and duration of intensive care unit/overall hospital stay was 2/10 days. At discharge, trace PVL, a peak/mean gradient of $34 / 20 \mathrm{~mm} \mathrm{Hg}$, and an effective opening area of $1.4 \mathrm{~cm}^{2}$ were documented in the aortic position. Regarding tricuspid $\mathrm{ViR}$, mild residual TR at the annuloplasty ring interruption was observed where the ventricular PM lead was still located with adequate lead function. At 30 days, the patient reported improvement in NYHA class II.

\section{Discussion and Conclusion}

Surgical redooperations for TR carry substantial perioperative risk, which is even higher in patients with previous bypass surgery and impaired right ventricular function. ${ }^{3-5}$ Conservative treatments failed and to avoid resternotomy or cardiopulmonary bypass-despite the knowledge of the asymmetric and partial landing zone provided by the open annuloplasty ringdecision was made to allocate this patient to a ViV/ViR intervention. An alternative strategy could be tricuspid valve clipping. However, tricuspid valve clipping was not an option due to anatomical reasons. Other alternative approaches such as annuloplasty devices were not applicable due to prior surgery. ${ }^{6}$

Despite technical success with substantial clinical benefit of this particular patient, some technical challenges should be briefly discussed. Abdoulhosn et al recently described an experience in 22 tricuspid ViR procedures with implantation success of $91 \%$ and significantly reduced degrees of TR (excluding PVLs). As in the present case, almost all tricuspid annuloplasty rings had an interrupted portion to respect the patients' conduction system anatomy. Abdoulhosn et al described significant residual PVLs at the open aspect of the 
ring in a subset of patients. As a solution, Amplatzer occluders were implanted into the residual paravalvular space. ${ }^{7}$

A further challenge lies in the patient's history of a twochamber PM implantation. The ventricular lead posed a risk of lead damage during the ViR procedure. In this case, the lead position was at the interrupted ring portion. For RVP, an external programmer was used. In case of any lead dysfunction, the placement of a temporary coronary sinus PM wire might have been an option. ${ }^{8}$

When using the 29-mm SAPIEN3 THV (Edwards Lifesciences, Inc.), it seemed advisable to perform loading of the THV onto the deployment balloon outside the eSheath to avoid friction of THV stent against the balloon, which might have led to balloon damage or disengagement of THV and balloon when advancing the system further through the eSheath.

This patient presented with only mild chronic renal failure. Thus, combined valvular intervention seemed feasible, especially since $\mathrm{ViV} / \mathrm{ViR}$ procedures rarely require increased amounts of contrast agent due to the radioopacity of prosthetic landing zones. In case of severe renal failure, a staged approach may be considered.

In conclusion, this case demonstrated the feasibility of concomitant transcatheter aortic ViV and tricuspid ViR procedures. Careful consideration of the above-mentioned technical challenges and anticipation of the high degree of procedural complexity are mandatory for such combined transcatheter valve interventions.

\section{References}

1 Phan K, Zhao DF, Wang N, Huo YR, Di Eusanio M, Yan TD. Transcatheter valve-in-valve implantation versus reoperative conventional aortic valve replacement: a systematic review. J Thorac Dis 2016;8(01):E83-E93

2 Conradi L, Silaschi M, Seiffert M, et al. Transcatheter valve-invalve therapy using 6 different devices in 4 anatomic positions: clinical outcomes and technical considerations. J Thorac Cardiovasc Surg 2015;150(06):1557-1565; 1567.e1-1567.e3; discussion, 1565-1567

3 Rogers JH, Bolling SF. The tricuspid valve: current perspective and evolving management of tricuspid regurgitation. Circulation 2009;119(20):2718-2725

4 Onorati F, Perrotti A, Reichart D, et al. Surgical factors and complications affecting hospital outcome in redo mitral surgery: insights from a multicentre experience. Eur J Cardiothorac Surg 2016;49(05):e127-e133

5 Boldt J, Zickmann B, Thiel A, Dapper F, Hempelmann G. Age and right ventricular function during cardiac surgery. J Cardiothorac Vasc Anesth 1992;6(01):29-32

6 Rodés-Cabau J, Hahn RT, Latib A, et al. Transcatheter Therapies for Treating Tricuspid Regurgitation. J Am Coll Cardiol 2016;67(15): 1829-1845

7 Aboulhosn J, Cabalka AK, Levi DS, et al. Transcatheter valve-inring implantation for the treatment of residual or recurrent tricuspid valve dysfunction after prior surgical repair. JACC Cardiovasc Interv 2017;10(01):53-63

8 Sideris S, Drakopoulou M, Oikonomopoulos G, et al. Left ventricular pacing through coronary sinus is feasible and safe for patients with prior tricuspid valve intervention. Pacing Clin Electrophysiol 2016;39(04):378-381 